\title{
MOTIVAÇÃO DE CRIANÇAS E ADOLESCENTES PRATICANTES DE ESPORTES EM PROJETOS DE INCLUSÃO SOCIAL DO RIO DE JANEIRO
}

\author{
Darla de Corvalho Barreto' \\ Rodrigo Silva Perfeito
}

\section{RESUMO}

O objetivo desta pesquisa visa analisar o efeito do clima motivacional e comportamental criado pelos professores de um projeto de inclusão social (PIS) por meio do esporte sobre a orientação de vida de seus alunos. Para esta análise foi utilizado o TEOSQ aplicado em 42 jovens de ambos os sexos, na faixa etária de 10 a 17 anos, que participam dos PIS oferecidos pela Secretaria de Esporte e Lazer de São João de Meriti e sua Vila Olímpica, nas modalidades de dança, futebol, jiu-jítsu e vôlei. Estes 42 foram classificados em dois grupos: iniciantes, até seis meses de prática, e veteranos, acima de seis meses até setenta e dois meses de prática. Os resultados obtidos sugerem que não houve diferença relevante entre as sub-escalas ego-tarefa dos grupos; concluindo que os PIS aparentam não influenciar os indivíduos na construção de valores, como prevenção da adesão a criminalidade a que são expostos.

Palavras-chave: Projetos de inclusão social. Motivação. Orientação de vida. TEOSQ

1 Graduada em Educação Física pela Universidade do Estado do Rio de Janeiro (UERJ). Rio de Janeiro/Rio de Janeiro, Brasil. E-mail: darlaohlavrac@gmail.com

2 Mestre em Ciências da Atividade Física. Diretor do Instituto Fisart (FISART). Rio de Janeiro/Rio de Janeiro, Brasil. E-mail: rodrigosper@yahoo.com.br 


\title{
MOTIVATION OF PRACTING CHILDREN AND ADOLESCENTS OF SPORTS IN PRO- JECTS OF SOCIAL INCLUSION OF RIO DE JANEIRO
}

\begin{abstract}
The point of this research is to analyze the effect in motivation and behavior, created by the social including project (SIP) professors using sport as a guide in theirs students life. For this analysis was used the TEOSQ applaied in 42 young people of both sex, aged 10 - 17 years that participates of SIP offered by the Department of Sport and Recreation in São João de Meriti and their Olympic Village, in modalities of dance, soccer, volleyball and jiu-jitsu. These 42 were classified in two groups: beginners, until six months of practice, and veterans, over six months until seventy-two months of practice. The results suggest no significant difference between the subscales ego-task groups; concluding that the SIPs do not appear to influence individuals in building values such as preventing adhesion to criminality that they are exposed.
\end{abstract}

Keywords: Social inclusion projects. Motivation. Life orientation. TEOSQ

\section{MOTIVACIÓN PARA NIÑOS Y ADOLESCENTES EN DEPORTOS EN SOCIAL PROYECTO DE INCLUSIÓN DE RIO DE JANEIRO}

\section{RESUMEN}

El objetivo de esta investigación es analizar el efecto del clima motivacional y conductual creado por los profesores de un proyecto de inclusión social (PIS) a través del deporte en el curso de la vida de sus estudiantes. Para este análisis se utilizó TEOSQ aplicado a 42 jóvenes de ambos sexos, con edades entre 10-17 años que participan en el PIS ofrecido por el Departamento de Deportes y Recreación de São João de Meriti y su Villa Olímpica, las formas de danza, fútbol, jujitsu y voleibol. Estos 42 fueron clasificados en dos grupos: principiantes, hasta seis meses de práctica, y veteranos, más de seis meses hasta setenta y dos meses en la práctica. Los resultados sugieren que no hubo diferencia significativa entre los grupos de auto-tarea subescalas; concluyendo que los PIS no parecen influir en los individuos en la construcción de valores, tales como la prevención de la delincuencia adhesión a los que están expuestos.

Palabras clave: Proyecto de inclusión social. La motivación. La vida de orientación. TEOSQ 


\section{INTRODUÇÃO}

Após 1980 houve um crescente número de projetos de inclusão social (PIS) que tinham como objetivo principal atender crianças e adolescentes apontados como sujeitos vulneráveis à pobreza, violência e exclusão social, com o intuito de educar e ocupar seu tempo ocioso (ZALUAR, 1994). Ação empreendida por vários setores, como o público, o privado, organizações não governamentais e também por pessoas famosas.

De acordo com Mezzadri et. al (2010), os PIS também são vistos como uma opção de lazer segura que propicia atividade física e esportiva, favorecendo a socialização e oportunizando perspectiva de profissionalização, que consequentemente, minimiza a exposição ao contato com a violência local e com as drogas.

Propicia o alcançar da dimensão simbólica do desenvolvimento humano, que para Branco (2016) é facilitado por práticas socioculturais que constroem contextos de interação social concretas entre os indivíduos.

Desta forma, a prática do esporte ou de atividades sócio-educativas como meio de socialização positiva ou inclusão social tem motivado a elevação do número de projetos esportivos destinados aos jovens das classes populares. Os objetivos dos projetos são orientados pela perspectiva da inclusão social e por proporcionar outros caminhos àqueles desfavorecidos pela falta da estruturação local (CASTRO e SOUZA, 2011).

Segundo Magill (1990) a motivação se relaciona com o motivo, sendo este o responsável por produzir o impulso inicial para um comportamento. Este é originalizado por necessidades internas, mas pode ser influenciado pelo meio ambiente. Folle e Texeira (2012) evidenciam que é necessário dar importância aos motivos por serem responsáveis por determinar as ações, sendo uma das causas para implementação dos projetos a falta de investimentos em locais economicamente desfavorecidos.

Um aspecto nem sempre observado nos PIS que utilizam o esporte como alternativa à socialização exercida pela criminalidade, - encontra-se na avaliação do impacto das atividades desenvolvidas e como estas influenciam na formação dos participantes - seja ela motora, social, moral ou psicológica (VIANNA e LOVISOLO, 2009b).

Os idealizadores parecem admitir que a formação positiva por meio do esporte seja a solução desta carência, sem perceber que este pode ser bem ou mal conduzido, resultando em formação positiva ou negativa de crianças e adolescentes. Se entendermos que a segunda alternativa está correta, destaca-se a necessidade de instrumentos e procedimentos que possam ser utilizados para avaliar até que ponto as intervenções desenvolvidas nos PIS estão contribuindo para a construção e afirmação de atitudes e comportamentos para a formação de um cidadão, fornecendo aos responsáveis e interventores informações para a elaboração de novas propostas de operação.

A análise motivacional da teoria de orientação de vida por objetivos oferece uma direção para pesquisas que procuram o entendimento e a explicação dos motivos subjacentes aos comportamentos no esporte. Essa teoria tem abordado os antecedentes e as consequências sociais, psicológicas e comportamentais de duas orientações de vida por objetivos independentes, chamadas orientação de vida Ego (orientação Ego) e orientação 
de vida (orientação Tarefa), que são definidas conforme a percepção subjetiva de sucesso e de fracasso na atividade (DUDA e NTOUMANIS, 2003).

Assim, esta investigação se propõe analisar o efeito do clima motivacional e comportamental criados pelos professores de um PIS por meio do esporte sobre a orientação de vida de seus alunos.

Possui sua relevância científica na tentativa de atribuir novos dados aos escassos artigos publicados na delimitação destacada. Já sua relevância social se estrutura na busca por auxiliar e estimular a melhoria das políticas de intervenção que fortaleçam e resgatem os valores sociais através do esporte, tanto no ensino, quanto na sua prática, diminuindo a lacuna do conhecimento a respeito deste assunto e favorecendo a construção e solidificação de uma cultura esportiva positiva como opção a uma situação conflituosa imposta pelo meio social degradado e violento.

\section{METODOLOGIA}

\section{Tipo de pesquisa e número da amostra}

Trata-se de um estudo descritivo de caráter exploratório com número amostral de 42 alunos de ambos os sexos, com idades entre 10 e 17 anos, matriculados nas modalidades esportivas de jiu-jítsu, futebol, dança e vôlei dos PIS oferecidos pela Secretaria de Esporte e Lazer da cidade de São João de Meriti e da sua Vila Olímpica situados no estado do Rio de Janeiro.

Os participantes foram divididos de acordo com o tempo de experiência em Iniciantes com até 6 meses de prática e Veteranos de 6 a 72 meses de prática.

O presente trabalho atendeu as normas para a realização de pesquisa em seres humanos, Resolução 196/96 do Conselho Nacional de Saúde de 10/10/1996 (BRASIL, 1996) e da Resolução de Helsinque de 1975 (WMA, 2008).

\section{Instrumento de coleta}

Para a coleta dos dados foram distribuídos aos participantes 115 questionários TEOSQ e o Termo de Consentimento Livre e Esclarecido. Deste total, 42 retornaram com o Termo assinado. Os participantes que não continham o documento assinado foram excluídos da pesquisa. Em dia posterior, os sujeitos remanescentes responderam ao questionário com 13 questões que mede as orientações de vida no esporte e uma anamnese para coleta de dados sociais, mantendo sua identidade em anonimato. Alguns dados foram alcançados por meio de ficha de matrícula e acompanhamento presentes no projeto. O período de coleta ocorreu do dia 19 a 23 de dezembro de 2016.

Vale a ressalva de que apesar da utilização da anamnese no processo de coleta de dados, não utilizamos os dados encontrados por apresentarem focos destenuantes do 
objeto de pesquisa e por demandar explicações de cunho sociológico e antropológico para explicar o conceito de herói, de vitória, de anti-bullying, entre outros, que afastaria o foco de nosso estudo. Portanto, citamos a anamnese mesmo sem utilizar na discussão e nos resultados, pois a mesma esteve presente no momento de coleta dos dados.

O TEOSQ ou Task and Ego Orientation in Sport é um questionário fechado composto por 6 questões de cunho da sub-escala Ego e 7 questões de sub-escala Tarefa. Portanto, testa através de escores as propriedades psicométricas, ou seja, a validade, fidedignidade e a reprodutibilidade das ações conduzidas por praticantes de esportes, possibilitando descrever se o nível de motivação da ação está mais voltado para a tarefa ou ego. Todas as questões possuem respostas em uma escala de motivação tipo Likert de cinco pontos (de 1 - Não concordo mesmo, a 5 - Concordo mesmo). O questionário foi validado por Feijó e Feijó (1997).

Este instrumento foi escolhido para a presente pesquisa por ser um questionário específico para atividades esportivas tais como as conduzidas nos projetos sociais, possibilitando o alcançar de informações importantes que auxiliam no entender dos motivos que os jovens se inserem e, principalmente, se mantêm matriculados nos projetos sociais que objetivam melhoras sociais e educacionais.

\section{Procedimentos para análise dos dados}

O emprego de técnicas da estatística descritiva visou caracterizar o universo amostral pesquisado. Para a descrição dos dados coletados foram utilizadas medidas de localização e de dispersão.

A estatística descritiva das médias aritméticas das duas sub-escalas (Ego e Tarefa) permitiu a determinação da orientação de vida dos sujeitos como sugere Levin (1987).

Visando elucidar o significado da expressão "orientação para tarefa" e "orientação para o ego", que serão utilizadas na discussão e nas tabelas dos resultados, segue uma breve explicação:

No ambiente do questionário indicado para a orientação para a tarefa existem 7 questões, possibilitando o escore mínimo de 7 pontos e o máximo de 35 pontos, já que os pontos variam de 1 a 5 diante da escala de Likert (Q2 + Q5 + Q7 + Q8 + Q10 + Q12 + Q13). A soma desse escore avalia o quão intensa é a orientação para a tarefa, ou seja, o quanto o participante está focado em realizar as tarefas do projeto com expectativa de êxito sem ser contaminado por atraentes externos, como se tornar um atleta famoso e rico.

No ambiente do questionário indicado para a orientação para o ego existem 6 questões, possibilitando o escore mínimo de 6 pontos e o máximo de 30 pontos (Q1 + Q3 + Q4 + Q6 + Q9 + Q11). A soma desse escore avalia o quão intensa é a orientação para o ego, ou seja, mensura se os praticantes estão sendo seduzidos por fatores que aumentem seu ego emocional e social, como ser reconhecido e bem tratado na comunidade em que vive. Um grande escore para o ego pode desnortear o objetivo socializador e educacional dos projetos sociais. 
maio/2018

\section{Resultados}

Através da anamnese e documentos de matrícula, foram estabelecidos o quantitativo de participantes quanto ao sexo, modalidade, experiência esportiva, participação em competições ou eventos e frequência absoluta (não houve faltas) e relativa (percentual de alunos absolutos), gerando as tabelas de 1 a 6 .

Tabela 1: Distribuição dos participantes - Iniciantes

\begin{tabular}{ccc}
\hline Masculino & Feminino & Modalidade \\
\hline 0 & 3 & Dança \\
10 & 1 & Futebol \\
5 & 0 & Jiu-Jítsu \\
0 & 1 & Vôlei \\
\hline
\end{tabular}

Tabela 2: Distribuição dos participantes - Veteranos

\begin{tabular}{ccc}
\hline Masculino & Feminino & Modalidade \\
\hline 0 & 1 & Dança \\
6 & 0 & Futebol \\
9 & 4 & Jiu-Jítsu \\
2 & 0 & Vôlei \\
\hline
\end{tabular}

Tabela 3: Participação de indivíduos em competições ou eventos

\begin{tabular}{ccc}
\hline Veteranos & Iniciantes & Modalidade \\
\hline 1 & 1 & Dança \\
5 & 7 & Futebol \\
11 & 2 & Jiu-Jítsu \\
2 & 1 & Vôlei \\
$19(86,3 \%)$ & $11(55 \%)$ & Total \\
\hline
\end{tabular}

Tabela 4: Frequência absoluta e relativa - Iniciantes

\begin{tabular}{ccc}
\hline Freq. Absoluta & Freq. Relativa & Modalidade \\
\hline 3 & $15 \%$ & Dança \\
11 & $55 \%$ & Futebol \\
5 & $25 \%$ & Jiu-Jitsu \\
1 & $5 \%$ & Volêi \\
\hline
\end{tabular}


Tabela 5: Frequência absoluta e relativa - Veteranos

\begin{tabular}{ccc}
\hline Freq. Absoluta & Freq. Relativa & Modalidade \\
\hline 1 & $4,55 \%$ & Dança \\
6 & $27,27 \%$ & Futebol \\
13 & $59,09 \%$ & Jiu-Jitsu \\
2 & $9,09 \%$ & Volêi \\
\hline
\end{tabular}

Tabela 6: Frequência absoluta e relativa - Total

\begin{tabular}{ccc}
\hline Freq. Absoluta & Freq. Relativa & Modalidade \\
\hline 4 & $9,52 \%$ & Dança \\
17 & $40,48 \%$ & Futebol \\
18 & $42,86 \%$ & Jiu-Jitsu \\
3 & $7,14 \%$ & Volêi \\
\hline
\end{tabular}

As análises dos dados obtidos na aplicação do TEOSQ apontaram nos grupos classificados como iniciantes médias de orientação de vida para a Tarefa, ligeiramente, maiores que a orientação de vida para o Ego, no entanto, diferença estatisticamente irrelevante. Conforme é observado na tabela 7.

Tabela 7: Média dos Iniciantes

\begin{tabular}{ccc}
\hline Média Tarefa & Média Ego & Indivíduo \\
\hline 4,86 & 3,83 & $1 \mathrm{~A}$ \\
5 & 3,67 & $2 \mathrm{~A}$ \\
4,86 & 3,33 & $3 \mathrm{~A}$ \\
2,49 & 3,83 & $4 \mathrm{~A}$ \\
4,43 & 3,17 & $5 \mathrm{~A}$ \\
5 & 3,5 & $6 \mathrm{~A}$ \\
2,86 & 1,83 & $7 \mathrm{~A}$ \\
3 & 1,83 & $8 \mathrm{~A}$ \\
3,29 & 2,33 & $9 \mathrm{~A}$ \\
4 & 3 & $10 \mathrm{~A}$ \\
4,29 & 1,83 & $11 \mathrm{~A}$ \\
4,43 & 4,33 & $12 \mathrm{~A}$ \\
4,43 & 2,83 & $13 \mathrm{~A}$ \\
\hline
\end{tabular}


maio/2018

\begin{tabular}{ccc}
\hline Média Tarefa & Média Ego & Indivíduo \\
\hline 5 & 2,33 & $14 \mathrm{~A}$ \\
4 & 3,33 & $15 \mathrm{~A}$ \\
4,57 & 3 & $16 \mathrm{~A}$ \\
4,29 & 3,67 & $17 \mathrm{~A}$ \\
4,86 & 3,17 & $18 \mathrm{~A}$ \\
4,14 & 3,33 & $19 \mathrm{~A}$ \\
4,43 & 4 & $20 \mathrm{~A}$ \\
\hline
\end{tabular}

Esses pequenos níveis maiores, porém estatisticamente sem diferença relevante para orientação de vida para a Tarefa (em relação à orientação para o Ego), também foram constatados nos dados advindos dos praticantes veteranos, como consta na tabela 8 .

Tabela 8: Média dos Veteranos

\begin{tabular}{ccc}
\hline Média Tarefa & Média Ego & Indivíduos \\
\hline 3,86 & 3,17 & $1 \mathrm{~B}$ \\
4,43 & 3 & $2 \mathrm{~B}$ \\
4,86 & 2,83 & $3 \mathrm{~B}$ \\
4,86 & 3,17 & $4 \mathrm{~B}$ \\
4,14 & 2 & $5 \mathrm{~B}$ \\
4,86 & 3 & $6 \mathrm{~B}$ \\
4,29 & 2,17 & $7 \mathrm{~B}$ \\
4,43 & 3,67 & $8 \mathrm{~B}$ \\
4,57 & 3,33 & $9 \mathrm{~B}$ \\
4,29 & 3,5 & $10 \mathrm{~B}$ \\
2,43 & 1,83 & $11 \mathrm{~B}$ \\
5 & 4,17 & $12 \mathrm{~B}$ \\
4,14 & 2,67 & $13 \mathrm{~B}$ \\
3,57 & 2,67 & $14 \mathrm{~B}$ \\
4,57 & 2,33 & $15 \mathrm{~B}$ \\
4,14 & 2,17 & $16 \mathrm{~B}$ \\
4,57 & 3,5 & $17 \mathrm{~B}$ \\
4,57 & 3,5 & $18 \mathrm{~B}$ \\
4 & 2,67 & $19 \mathrm{~B}$ \\
4,71 & 2,83 & $20 \mathrm{~B}$ \\
4,57 & 3,33 & $21 \mathrm{~B}$ \\
4,86 & 3,17 & $22 \mathrm{~B}$ \\
\hline & & \\
\hline & 33 & 3 \\
\hline
\end{tabular}


Notou-se, através das médias apresentadas na tabela 9, que tanto o grupo dos veteranos, quanto o dos iniciantes, apresentaram orientação motivacional de vida para a Tarefa com médias de 4,3 para os iniciantes e 4,35 para os veteranos. Quando se compara os níveis apresentados em cada grupo para as duas sub-escalas, o grupo dos veteranos sugere não apresentar diferenças relevantes, visto que os valores apresentados nas duas sub-escalas dos dois grupos são ligeiramente próximas. Na sub-escala Ego a diferença foi de 0,17 a mais para os veteranos e na sub-escala Tarefa o número encontrado foi de 0,05 a mais para os mesmos.

Tabelas 9: Médias dos Escores do TEOSQ dos grupos Iniciantes e Veteranos

\begin{tabular}{ccc}
\hline Iniciantes & Veteranos & Sub-escala \\
\hline 3,11 & 2,94 & Ego \\
4,3 & 4,35 & Tarefa \\
\hline
\end{tabular}

\section{Discussão}

A presente pesquisa pretendeu estudar a orientação motivacional que crianças e adolescentes recebem ao praticarem esportes através dos PIS, averiguando sua interferência nos processos motivacionais, que por sua vez, busca diminuir a probabilidade do aumento da orientação motivacional para o Ego (MIRANDA et. al, 2012). Também é possível constatar a transferência de valores culturais através do desporto, sendo os agentes socializadores os principais responsáveis pela qualidade desse ambiente (RAPOSO, 2005, AQUINO, 2016).

Os resultados obtidos pelo perfil motivacional dos grupos Iniciantes e Veteranos apontam que não há diferença relevante entre as sub-escalas (para que houvesse um resultado positivo a favor dos projetos, a escala tarefa deveria ter um escore significativamente maior do que a escala ego, fato que não ocorreu), mostrando a possível baixa do impacto esperado em sua orientação motivacional, sugerindo que os objetivos que os PIS se propõem não estão sendo alcançados, colocando em dúvida, portanto, se os valores sociais apontados por Castro e Souza (2011) junto à prática esportiva estão realmente ocorrendo.

Esses dados que comprovariam a possível falha motivacional abordada nos PIS, podem ser analisados por diversas vertentes. Uma delas seria a influência da configuração sócio-cultural na qual esses indivíduos estão inseridos, podendo interferir na leitura dos resultados obtidos. Conforme Vianna e Lovisolo (2009a) exemplificam, as propostas dos PIS devem interagir com os indivíduos e sua localidade. E caso não ocorra essa especificidade, o projeto fica mais vulnerável a erros.

Em outras palavras, os indivíduos que residem em São João de Meriti são culturalmente influenciados pela configuração social local, visto que a variância encontrada foi de 0,05 entre os iniciantes e os veteranos. Se este mesmo teste fosse aplicado em um PIS localizado em um local privilegiado da cidade do Rio de Janeiro, a probabilidade de encontramos percentuais diferentes seria bastante alta. E por isso a importância de não 
aplicar somente o esporte como modalidade de movimento, mas sim aproximar o máximo possível da especificidade cultural dessas pessoas.

Perfeito (2011) explica essa necessidade diante das representações simbólicas, em que cada indivíduo constrói de modo individualizado suas percepções de vida moldados por ideais, teias de relacionamento, emoções, culturas, entre outros, fazendo com que cada comportamento e a percepção de vida sejam extremamente influenciados pela cultura local.

Outro fator importante para o possível insucesso dos PIS é o tempo que cada criança ou adolescente passa efetivamente participando e praticando esportes. Assim, a proximidade das médias encontradas neste trabalho, tanto quando se compara as duas sub-escalas, quanto na diferença entre os Iniciantes e Veteranos pode, como explica Vianna e Lovisolo (2009a), ter sido influenciado pelo baixo tempo de participação e envolvimento dos participantes nos PIS, como por faltas e ausências de longos períodos (tabelas 4, 5 e 6). Portanto, é preciso criar estratégias que diminuam a evasão dessas crianças e adolescentes, e de novo, a aproximação das atividades para a cultura da localidade poderia ajudar.

Diante dos dados encontrados nas fichas dos participantes e nos escores do TEOSQ, a competição é um dos motivos mais expressivos para a prática esportiva, sendo de intensidade média para os Iniciantes e alta para os Veteranos. Estes dados, trazem respostas diferentes das esperadas, que para Caruzzo et al. (2013) seria a fidelização do praticante por construção de comportamento social positivo, como a importância da ajuda ao outro ou do respeito as regras, ou ainda, para Bezerra e Perfeito (2013) a mudança de comportamento e a perspectiva de um futuro melhor.

Salienta-se que a orientação para o Ego deve ser evitada para minimizar ações que não vão de encontro aos valores sociais, fator este que aumenta possíveis vulnerabilidades desses jovens principalmente pela baixada fluminense vivenciar relatos do aumento da violência (MIAGUSKO, 2016; SILVA, 2016), pela hipótese da migração de bandidos das comunidades da cidade do Rio de Janeiro com a instalação das UPPs.

Em últimas palavras, se existe uma tendência nacional de se pensar que os PIS estimulam seus participantes por meio da orientação motivacional para tarefa como meio de potencializar valores que beneficie sua formação social e minimização dos possíveis riscos da violência aos quais crianças e jovens são expostos, é preciso repensar se estes são apenas dados ou a realidade dessas áreas carentes.

Como vimos na tabela 3, nesse PIS, boa parte dos matriculados, principalmente na modalidade do futebol, buscam competições através do programa social. É preciso averiguar através de novas pesquisas, uma vez que esta se resume ao objetivo e delimitação destacados, quais são as mudanças sociais e comportamentais que essa variável pode causar na vida dessas crianças e adolescentes, uma vez que a competição mal orientada pode estimular efeitos antagônicos ao proposto pelos projetos, que é, por exemplo, a cooperação.

\section{CONCLUSÃO}

O resultado obtido neste estudo mostra que os dados apontados na orientação motivacional das sub-escalas, apresentam diferenças relativamente baixas ao se comparar 
a orientação motivacional para o ego com a orientação motivacional para a tarefa do grupo classificado como veterano e sua sub-escala motivacional para a tarefa com as do grupo dos iniciantes.

Através destes achados, verificou-se que o PIS parece não surtir efeito sobre a orientação motivacional das crianças e adolescentes praticantes de esportes, deixando questionamento sobre a influência do PIS no processo de formação social dos participantes.

Devido ao reduzido número de sujeitos que foram analisados, propõem-se a elaboração de novos estudos com um número maior de participantes e com comparações entre economia, cultura, sexo e modalidades esportivas diferentes.

\section{REFERÊNCIAS}

AQUINO, G. O esporte como elemento socializador e formador de crianças e jovens. Faminas, v. 6, n. 2, 2016.

BEZERRA, M; PERFEITO, R. Variação do humor por meio de exercícios de Pilates em adolescentes acautelados. Nova Fisio. n. 90, jan/fev, 2013.

BRANCO, A. Crenças e práticas culturais: co-construção e ontogênese de valores sociais. Pro-posições, v. 17, n. 2, p. 139-155, 2016.

BRASIL. Normas para a Realização de Pesquisas em Seres humanos. C.N.D. Saúde. Resolução 196/96, 1996.

CARUZZO, N; NASCIMENTO JUNIOR, J; VIEIRA, J; VIEIRA, L. Orientação de metas no contexto do vôlei de praia: um estudo comparativo entre atletas medalhistas e não medalhistas. Revista brasileira de ciência e movimento, v. 21, n. 3, p. 42-50, jun, 2013.

CASTRO, S; SOUZA, D. Significado de um projeto social esportivo: um estudo a partir das perspectivas de profissionais, pais, crianças e adolescentes. Movimento. Porto Alegre, v. 17, n. 04, p. 145-163, out/dez, 2011.

DUDA, J; NTOUMANIS, N. Correlates of achievement goal orientations in physical education. International Journal of Educational Research, v. 39, n. 4-5, p. 415-436, 2003.

FEIJÓ, O; FEIJÓ, M. Validação Brasileira da Bateria de Testes da Dra. Joan Duda e Associados Sobre Motivação Para a Prática Do Esporte. Relatório Final de Pesquisa ao CNPq. Brasília, 1997.

FOLLE, A; TEXEIRA, F. Motivação de escolares das séries finais do Ensino Fundamental nas aulas de Educação Física. Rev. Educ. Fís/ UEM, v. 23, n. 1, p. 37-44, jan-fev- mar, 2012.

LEVIN, J. Estatística aplicada a ciências humanas. 2 ed. São Paulo: Harbra, 1987.

MAGUILL, R. Aprendizagem Motora: Conceitos e aplicações. São Paulo: Edgar Blücher, 1990.

MEZZADRI, F. M. et. al, Determinantes para a implantação de um projeto social. Motriz, Rio Claro, v. 16, n. 03, p. 689-700, jul/set, 2010.

MIAGUSKO, E. Esperando a UPP. Circulação, violência e mercado político na Baixada Fluminense. Revista Brasileira de Ciências Sociais, v. 31, n. 91, p. 1-15, 2016. 
maio/2018

MIRANDA, R. et al. O fluxo no voleibol: relação com a motivação, autoeficácia, habilidade percebida e orientação às metas. Rev. Educ. Fis/UEM, São Paulo, v. 23, n. 3, p. 379387, Jul- Agos-Set, 2012.

PERFEITO, R. A Educação Física e o Bullying: a desutilização da inteligência. Livre Expressão: Rio de Janeiro, 2011.

RAPOSO, J. et. al. Caracterização dos níveis de negativismo, ativação, autoconfiança e orientações motivacionais de alpinistas. Motricidade, v. 5, n. 2, p. 63-86, jun, 2009.

SILVA, L. Baile Funk, missão civilizatória e UPP: cultura e segurança pública na cidade do Rio de Janeiro. Brasiliana-Journal for Brazilian Studies, v. 4, n. 2, p. 318-342, 2016.

VIANNA, J; LOVISOLO, H. Projetos de inclusão social através do esporte: notas sobre a avaliação. Movimento, Porto Alegre, v. 15, n. 3, p. 145-162, jul./set, 2009a.

VIANNA, J; LOVISOLO, H. Desvalorização da aprendizagem técnica na Educação Física: evidências e críticas. Motriz, Rio Claro, v. 15, n. 4, p. 883-889, out/dez, 2009b.

ZALUAR, A. Cidadãos não vão ao paraíso: juventude e política social. Rio de Janeiro: Escuta, 1994.

WMA. WORLD MEDICAL ASSOCIATION. Declaration of Helsinki. Ethical Principles for Medical Research Involving Human Subjects. 59th WMA General Assembly, Seoul, October, 2008.

Recebido em: Março/2017 Aprovado em: Agosto/2017 